\title{
Análise geográfica da distribuição dos serviços de saúde de Terapia Intensiva para atendimentos dos casos de COVID-19 no Rio Grande do Sul, Brasil
}

\author{
GASS, Sidnei Luís Bohn'; SILVA, Dieison Morozoli da²
}

1Professor da Universidade Federal do Pampa - Campus Itaqui, e do Programa de Pós-Graduação em Geografia da UFRGS. Coordenador do projeto SIGPampa - sidneigass@unipampa.edu.br http://orcid.org/0000-0001-5197-7506

${ }^{2}$ Discente do Curso de Engenharia Cartográfica e de Agrimensura da Universidade Federal do Pampa Campus Itaqui - dieison.ufp@gmail.com - http://lattes.cnpq.br/0116359981757404

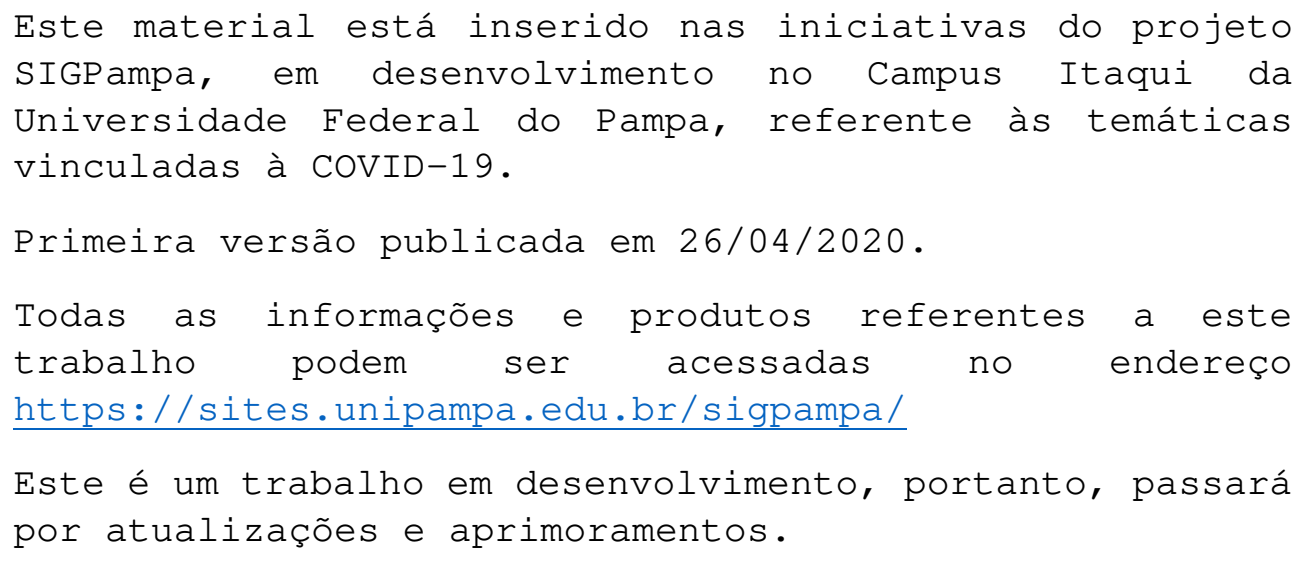




\section{RESUMO}

Dando continuidade aos esforços do projeto SIGPampa, este trabalho direciona seus esforços para a caracterização da estrutura de assistência avançada dos serviços de saúde do Estado do Rio Grande do Sul. A distribuição espacial pode ser relacionada às causas de doenças, bem como a distribuição geográfica dos serviços de saúde pode ser decisiva para a execução de atendimentos e tomada de decisões. Com isso, o objetivo deste trabalho foi apresentar uma análise geográfica da distribuição dos serviços de saúde de terapia intensiva e disponibilidade de ambulâncias de suporte avançado no Rio Grande do Sul. Para tanto, foi realizado um recorte analítico das macrorregiões de saúde do estado e suas subdivisões. Foram utilizados dados correspondentes a projeções populacionais para o ano de 2019, além de dados oficiais relacionados a pandemia. Os dados foram trabalhados através de planilhas eletrônicas e o software QGIS 3.10.3 Foram apresentados os números correspondentes a quantidade de leitos UTI SUS, UTI SUS direcionadas para atendimento aos casos de COVID-19, bem como demais indicadores referentes a capacidade de atendimentos correspondentes a cada um dos domínios de atendimentos de saúde. Ressalta-se a baixa disponibilidade de leitos, bem como a necessidade de ambulâncias de suporte avançado. Ainda em tempo, se questiona a distribuição não-linear da quantidade de leitos por número de habitantes.

\section{ABSTRACT}

Continuing the efforts of the SIGPampa project, this work directs its efforts towards the characterization of the advanced assistance structure of health services in the State of Rio Grande do Sul. The spatial distribution can be related to the causes of diseases, as well as the geographical distribution of Health services can be decisive for the execution of care and decision making. Thus, the objective of this work was to present a geographic analysis of the distribution of intensive care health services and the availability of advanced support ambulances in Rio Grande do Sul. For this purpose, an analytical analysis of the state's health macro-regions and their subdivisions. Data corresponding to population projections for the year 2019 were used, in addition to official data related to the pandemic. The data were worked through electronic spreadsheets and the QGIS 3.10.3 software. The numbers corresponding to the number of beds ICU SUS, SUS ICU directed to attend COVID-19 cases were presented, as well as other indicators referring to the attendance capacity corresponding to each of the health care domains. The low availability of beds is highlighted, as well as the need for advanced support ambulances. Still in time, the non-linear distribution of the number of beds per number of inhabitants is questioned. 


\section{INTRODUÇÃO}

Dando seguimento às pesquisas que o projeto SIGPampa vem desenvolvendo com relação à COVID-19 no Rio Grande do Sul (GASS \& DA SILVA, 2020), foi direcionado o olhar para a estrutura de assistência avançada dos serviços de saúde disponíveis no estado. A conjuntura que a COVID-19 (WIKIPEDIA, 2020a, 2020b, 2020c) vem impondo à sociedade nos seus mais variados campos de articulação e atuação, faz com que sejam necessárias tomadas de decisão em curto e médio prazo, processo este com o qual as estruturas públicas enfrentam dificuldades para lidar.

O espaço geográfico sempre apresentou alguma relação com as eventualidades epidemiológicas, como já mencionaram Gass \& da Silva (2020). De acordo com Bonfim \& Medeiros (2008), já no século XIX foi demonstrado que a distribuição espacial dos casos de cólera estava relacionada com a localização de uma bomba pública de água e sendo assim, o espaço foi um importante ponto para a inferências sobre o comportamento dos casos de doença. Contudo, para além da distribuição dos casos da COVID-19, há que se compreender de qual forma os serviços de saúde (neste caso, os leitos de UTI e as ambulâncias de suporte avançado) estão geograficamente distribuídos no território que por eles deve ser atendido. Esta é uma informação que, em última análise, pode ser denominada de estratégica, visto que tem a função de instrumentalizar a tomada de decisões quanto à gestão da pandemia.

Com o intuito de organizar as medidas a serem adotadas conforme o avanço da pandemia no âmbito do território do Rio Grande do Sul, a Secretaria da Saúde elaborou o Plano de Contingencia Hospitalar (SES-RS, 2020), que tem como objetivo principal orientar a rede de assistência à saúde quanto aos leitos hospitalares disponíveis e ampliados, distribuídos nas macrorregiões de saúde do RS.

Tomando por base o referido documento, além de outros dados mencionados no item materiais e métodos, o presente artigo tem por objetivo apresentar uma análise geográfica da distribuição dos serviços de saúde de terapia intensiva e disponibilidade de ambulâncias de suporte avançado no Rio Grande do Sul.

\section{RECORTE ANALÍTICO}

O presente estudo toma por base as Macrorregiões de Saúde do estado do Rio Grande do Sul. Contudo, cabe salientar que, para fins de organização e gestão da saúde, o território do Rio Grande do Sul está dividido em Macrorregiões, Coordenadorias Regionais de Saúde e Regiões de Saúde, como demonstrado pelo mapa da figura 1. Esta organização considera agrupamentos de municípios, tomando por base a dimensão dos seus territórios e, por conseguinte, as distâncias a serem percorridas. Em boa parte dos casos, os limites das Coordenadorias Regionais e das Regiões são coincidentes, havendo a subdivisão de uma Coordenadoria Regional em mais de uma Região quando o número de municípios a serem atendidos foi maior. A tabela 1 apresenta uma quantificação da população, número

\footnotetext{
${ }^{1}$ Unidade de Terapia Intensiva.
} 
de Coordenadorias Regionais de Saúde, de Regiões de Saúde e de municípios abrangidos por cada Macrorregião, conforme informações de SES-RS (2013).

Figura 1 - Organização da saúde no território do estado do Rio Grande do Sul

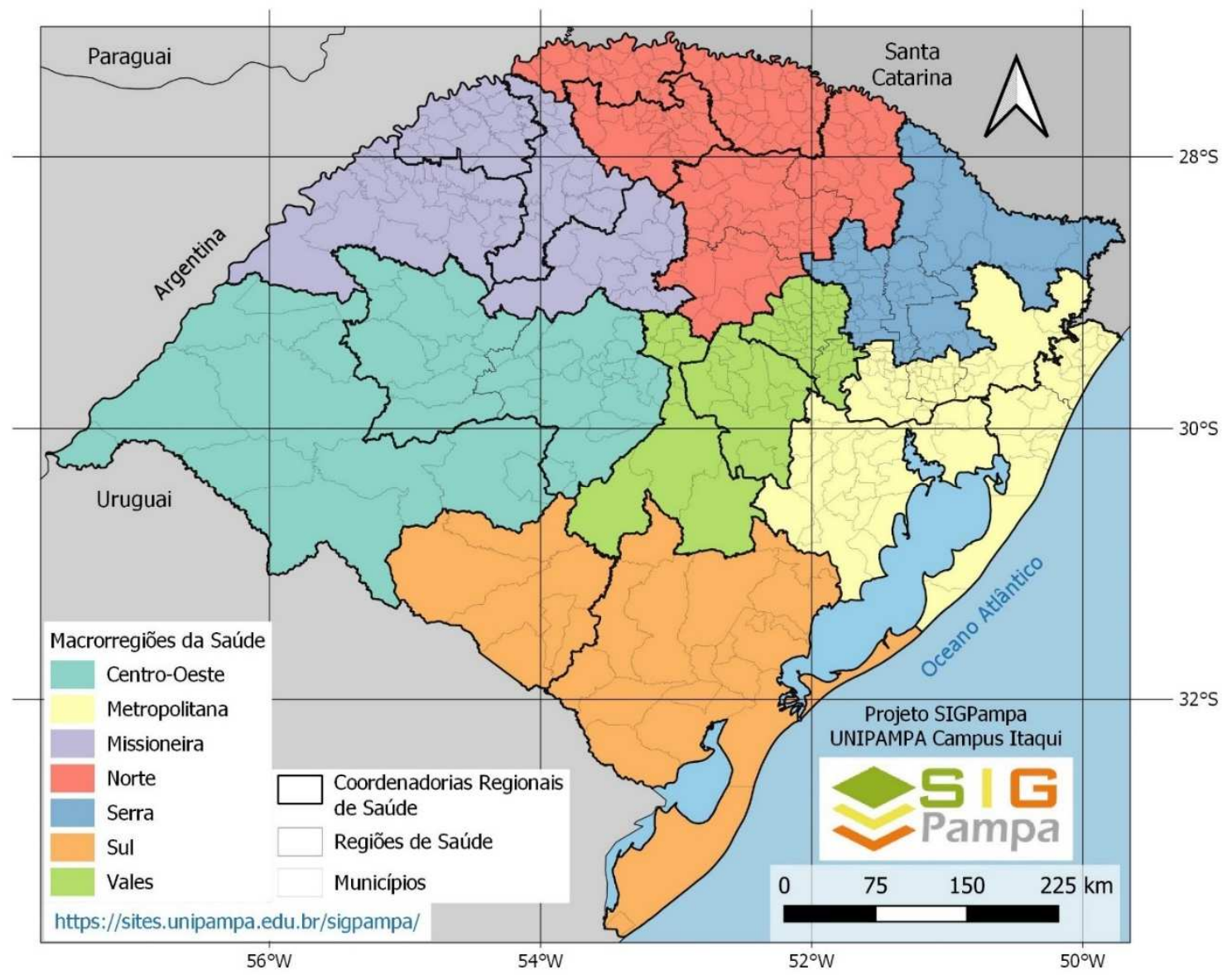

Fonte: elaborado pelos autores a partir de IBGE $(2017,2018)$ e SES-RS (2013)

Tabela 1 - Quantificação do número de Coordenadorias Regionais de Saúde, Regiões de Saúde e municípios, por Macrorregião de Saúde

\begin{tabular}{|c|c|c|c|c|c|}
\hline \multirow{2}{*}{ MACRORREGIÃO } & \multicolumn{2}{|c|}{ POP 2019} & \multirow{2}{*}{ CRS } & \multirow{2}{*}{ REG } & \multirow{2}{*}{ MUNIC } \\
\hline & \begin{tabular}{l|l} 
TOTAL \\
\end{tabular} & $\%$ & & & \\
\hline Centro-Oeste & 1017912 & 8.95 & 2 & 3 & 43 \\
\hline Metropolitana & 5023201 & 44.15 & 3 & 7 & 89 \\
\hline Missioneira & 884688 & 7.78 & 4 & 4 & 79 \\
\hline Norte & 1245819 & 10.95 & 4 & 6 & 147 \\
\hline Serra & 1227667 & 10.79 & 1 & 4 & 49 \\
\hline Sul & 1067296 & 9.38 & 2 & 2 & 28 \\
\hline Vales & 910656 & 8.00 & 3 & 4 & 62 \\
\hline RIO GRANDE DO SUL & 11377239 & 100.00 & 19 & 30 & 497 \\
\hline \multicolumn{6}{|c|}{$\begin{array}{l}\text { POP } 2019 \text { - população estimada para o ano de } 2019 \\
\text { CRS - Coordenadorias Regionais de Saúde } \\
\text { REG - Regiões de Saúde } \\
\text { MUNIC - municípios }\end{array}$} \\
\hline
\end{tabular}

Fonte: elaborado pelos autores a partir de IBGE (2019) e SES-RS (2013) 


\section{MATERIAIS E MÉTODOS}

Para o desenvolvimento do presente estudo foram utilizadas planilhas de cálculo eletrônico para a sistematização dos dados e geração das estatísticas básicas e, para a elaboração dos mapas, foi utilizada a plataforma QGIS 3.10.3, que é a principal ferramenta de trabalho do projeto SIGPampa.

Os dados utilizados são oriundos das seguintes fontes:

Dados populacionais da estimativa da população residente para o ano de 2019, sistematizados a partir do IBGE (2019);

Os quantitativos de leitos de UTI do SUS ${ }^{2}$ disponíveis, sua reserva para a COVID19, ampliação em função da COVID-19, foram extraídos do Plano de Contingência Hospitalar do Rio Grande do Sul (SES-RS, 2020);

As informações referentes aos leitos de UTI não SUS, e a disponibilidade de ambulâncias de suporte avançado, foram extraídas do Cadastro Nacional de Estabelecimentos de Saúde, através da plataforma DATASUS-TABNET (DATASUS, 2020), tendo com data de referência o mês de fevereiro de 2020;

Malha Territorial Municipal, compatível com a escala 1:250.000, em sua versão 2018 (IBGE, 2018).

Para chegar aos resultados apresentados neste artigo, foram executados os seguintes procedimentos metodológicos:

Organização dos dados considerando as Macrorregiões de Saúde do Rio Grande do Sul;

Geração das estatísticas básicas;

Inserção dos dados nas tabelas de atributos para espacialização dos dados;

Geração dos mapas temáticos;

Análise e discussão dos dados.

\section{ANÁLISE DOS DADOS}

A partir do Plano de Contingência Hospitalar (SES-RS, 2020), a Secretaria da Saúde traça um panorama de como poderá ocorrer a ampliação dos leitos de UTI para atender especificamente os casos de COVID-19 que necessitem deste tipo de suporte. O Plano prevê que 30\% dos leitos de Terapia Intensiva mantidos pelo SUS, existentes, (301 leitos de acordo com a Tabela 2), serão reservados para o atendimento de pacientes da COVID19. À medida em que os níveis ${ }^{3}$ estabelecidos pelo Plano forem avançando, há a previsão

\footnotetext{
2 Sistema Único de Saúde.

${ }^{3}$ Conforme mencionado pelo próprio Plano de Contingência Hospitalar (SES-RS, 2020), "a estruturação da resposta em três níveis é geralmente usada em planos de preparação e resposta em todo o mundo". Os níveis definidos pelo Plano, para o Rio Grande do Sul, são os seguintes:

Nível 0 - tendo como Indicador casos suspeitos, ainda sem confirmação.

Nível 1 - tendo como indicador até 500 internações suspeitas de COVID-19.

Nível 2 - tendo como indicador entre 500 a 1000 internações em leitos clínicos e de UTI com suspeita de COVID-19.
} 
da ampliação dos leitos, ou seja, o incremento de leitos novos, para além daqueles disponíveis.

Nos dados apresentados na Tabela 2, coluna "D", foi considerado o nível 3, que, de acordo com o Plano de Contingência considera como indicador, entre 1000 a 3000 internações em leitos clínicos e de UTI com suspeita de COVID-19, para o qual haveria um total de 872 leitos de UTI adulto disponíveis, sendo 301 leitos da contingência original, mais a ampliação de 571 leitos.

Contudo, os números absolutos apresentados, para que tenham uma representatividade dentro daquilo que a $\mathrm{OMS}^{4}$ estabelece, devem ser relativizados, considerando o quociente relativo a cada 10.000 habitantes. Neste sentido, antes da pandemia da COVID-19, a OMS recomendou que cada país tenha de 1 a 3 leitos de terapia intensiva para cada 10.000 habitantes. De acordo com o comunicado da Associação de Medicina Intensiva Brasileira (AMIB, 2020),

\begin{abstract}
segundo recomendações da OMS e do Ministério da Saúde, a relação ideal de leitos de UTI é de 1 a 3 leitos de UTI para cada 10 mil habitantes. Hoje, o SUS tem 1 leito para cada 10 mil habitantes, com pouca margem para aumento de demandas devido à alta taxa de ocupação. Na rede particular, a relação é de 4 leitos para cada 10 mil habitantes e a ocupação média é de $80 \%$.
\end{abstract}

Tomando por base estes quocientes, são apresentados na Tabela 2, os leitos disponíveis para cada 10.000 habitantes, por Macrorregião de Saúde, nos seguintes casos:

A - número total de leitos de UTI mantidos pelo SUS, antes do início da pandemia;

B - 30\% dos leitos de UTI mantidos pelo SUS antes da pandemia, reservados para pacientes de COVID-19, conforme Plano de Contingência Hospitalar (SES-RS, 2020);

C - número total de leitos de UTI não mantidos pelo SUS (particulares);

D - número total de leitos ampliados, considerando o nível 3 do Plano de Contingencia Hospitalar;

E - leitos de UTI mantidos pelo SUS, para cada 10.000 habitantes, antes da pandemia;

F - leitos de UTI mantidos pelo SUS, mais leitos UTI não mantidos pelo SUS, para cada 10.000 habitantes, antes da pandemia;

G - leitos de UTI para cada 10.000 habitantes, considerando os leitos dos grupos B e D;

$\mathrm{H}$ - leitos de UTI para cada 10.000 habitantes, considerando os leitos dos grupos $\mathrm{B}$, C e D.

Nível 3 - tendo como indicador entre 1000 a 3000 internações em leitos clínicos e de UTI com suspeita de COVID-19.

${ }^{4}$ Organização Mundial da Saúde. 
Tabela 2 - Dados populacionais, leitos de UTI em diferentes estimativas, número de ambulâncias e municípios por Macrorregião de Saúde do Rio Grande do Sul

\begin{tabular}{|c|c|c|c|c|c|c|c|c|c|c|c|}
\hline \multirow{2}{*}{ MACRORREGIÃO } & \multirow{2}{*}{$\begin{array}{l}\text { POP } \\
2019\end{array}$} & \multicolumn{8}{|c|}{ LEITOS UTI } & \multirow{2}{*}{ AMB } & \multirow{2}{*}{ MUNIC } \\
\hline & & $\mathbf{A}$ & B & C & D & $\mathbf{E}$ & $\mathbf{F}$ & G & $\mathrm{H}$ & & \\
\hline Centro-Oeste & 1017912 & 78 & 23 & 94 & 57 & 0.77 & 1.69 & 0.79 & 1.71 & 5 & 43 \\
\hline Metropolitana & 5023201 & 534 & 163 & 321 & 268 & 1.06 & 1.70 & 0.86 & 1.50 & 11 & 89 \\
\hline Missioneira & 884688 & 66 & 20 & 39 & 52 & 0.75 & 1.19 & 0.81 & 1.25 & 6 & 79 \\
\hline Norte & 1245819 & 87 & 25 & 58 & 46 & 0.70 & 1.16 & 0.57 & 1.04 & 5 & 147 \\
\hline Serra & 1227667 & 71 & 21 & 79 & 42 & 0.58 & 1.22 & 0.51 & 1.16 & 2 & 49 \\
\hline Sul & 1067296 & 113 & 34 & 39 & 54 & 1.06 & 1.42 & 0.82 & 1.19 & 5 & 28 \\
\hline Vales & 910656 & 52 & 15 & 15 & 52 & 0.57 & 0.74 & 0.74 & 0.90 & 8 & 62 \\
\hline RIO GRANDE DO SUL & 11377239 & 1001 & 301 & 645 & 571 & 0.88 & 1.45 & 0.77 & 1.33 & 42 & 497 \\
\hline \multicolumn{12}{|c|}{$\begin{array}{l}\text { POP } 2019 \text { - população estimada para o ano de } 2019 \\
\text { LEITOS UTI: } \\
\text { A - leitos UTI SUS } \\
\text { B - leitos UTI SUS 30\% para COVID-19 } \\
\text { C - leitos UTI não SUS } \\
\text { D - ampliação de leitos para o nível } 3 \text { do plano de contingência } \\
\text { E - leitos UTI SUS para cada } 10000 \text { habitantes } \\
\text { F - leitos UTI sus mas não SUS para cada } 10000 \text { habitantes } \\
\text { G - leitos UTI para cada } 10000 \text { habitantes (B+D) } \\
\text { H - leitos UTI para cada } 10000 \text { habitantes (B+C+D) } \\
\text { AMB - ambulâncias de suporte avançado disponíveis }\end{array}$} \\
\hline
\end{tabular}

Fonte: elaborado pelos autores a partir de IBGE (2019), SES-RS (2020) e DATASUS (2020)

De acordo com os dados apresentados na Tabela 2, o melhor cenário ocorre quando são somados os leitos de UTI mantidos pelo SUS mais àqueles não mantidos pelo SUS (particulares), ficando, neste caso, abaixo do índice mínimo de leitos sugeridos pela OMS, apenas a Macrorregião dos Vales. Contudo, a análise a ser apresentada pautar-se-á sobre os leitos do SUS.

Para uma compreensão especializada dos dados da Tabela 2, foram gerados mapas temáticos que permitem algumas análises. No mapa da Figura 2, é possível observar a distribuição da população em função das Macrorregiões. Neste mapa fica evidente a concentração populacional na Macrorregião Metropolitana, com mais de $40 \%$ do total do estado, seguida das Macrorregiões Serra e Norte.

No mapa da Figura 3, apresenta-se a distribuição dos leitos do SUS, para cada 10.000 habitantes, antes da pandemia. Comparando os mapas das Figuras 2 e 3, percebe-se um primeiro descompasso entre o quantitativo populacional e os leitos disponíveis. Se observarmos, por exemplo, o que ocorre com as Macrorregiões Missioneira, Norte e Serra, há uma inversão entre população e leitos disponíveis por habitante.

Ao se representar, no mapa da Figura 4, os leitos de UTI disponíveis no nível 3 do Plano de Contingência Hospitalar, o quadro que se apresenta podem gerar certa preocupação. Nas Macrorregiões Norte e Serra, que somam mais de $21 \%$ da população do estado, de acordo com a Tabela 1, apresentam um índice médio de leitos para cada 10.000 habitantes, de 0,54 , ao passo que a Macrorregião Missioneira, com 7,78\% da população do estado, tem uma disponibilidade de 0,81 leitos para cada 10.000 habitantes. 
Há que se considerar que, em função dos baixos índices de leitos por 10.000 habitantes disponíveis no estado, haverá pacientes que necessitem de remoção de um Estabelecimento de Saúde para outro. Neste caso, o transporte precisará ser realizado com ambulâncias de suporte avançado, ou seja, UTIs móveis, equipadas com respiradores/ventiladores. Neste caso, como representado pelo mapa da Figura 5, a Macrorregião da Serra possui o menor número de ambulâncias disponíveis, ao passo que as Macrorregiões Metropolitana e Vales possuem os melhores números.

Um elemento que deve ter espaço nas análises que são feitas, não apenas por esta pesquisa, mas, em especial naquelas que se debruçam sobre os protocolos de atendimento da COVID-19 e da gestão do sistema de saúde, é o fato de que

o coronavírus pode ter infectado quatro vezes mais pessoas no Rio Grande do Sul do que indicam os números oficiais. Esta é a projeção da pesquisa coordenada pela Universidade Federal de Pelotas (UFPel), que conta com a participação de pesquisadores da Universidade Federal do Pampa (Unipampa).

A primeira fase do estudo, realizada nos dias 11 e 12 de abril, mostra que para cada caso confirmado existem outros quatro não notificados. De acordo com a análise é de que 5.650 pessoas tenham sido infectadas pelo coronavírus no estado. Nesta quinta-feira, 16 de abril, o número de casos confirmados pela secretaria estadual da saúde não chega a 800. (UNIPAMPA, 2020)

Os dados da referida pesquisa, se associados ao fato de que no estado está ocorrendo a passagem do outono para o inverno, pode trazer à lume outra preocupação, que são as doenças respiratórias que se manifestam neste período. Como é amplamente conhecido, estas doenças podem ter efeitos mais significativos nos grupos de risco, que se assemelham aos da COVID-19. Com isto, pode haver um aumento de demanda por atendimento nos Estabelecimentos de Saúde, inclusive, em UTI. Cabe, no entanto, a estudos futuros esta análise. 
Figura 2 - Distribuição da população residente estimada para o ano de 2019, no Rio Grande do Sul, considerando as Macrorregiões de planejamento da saúde

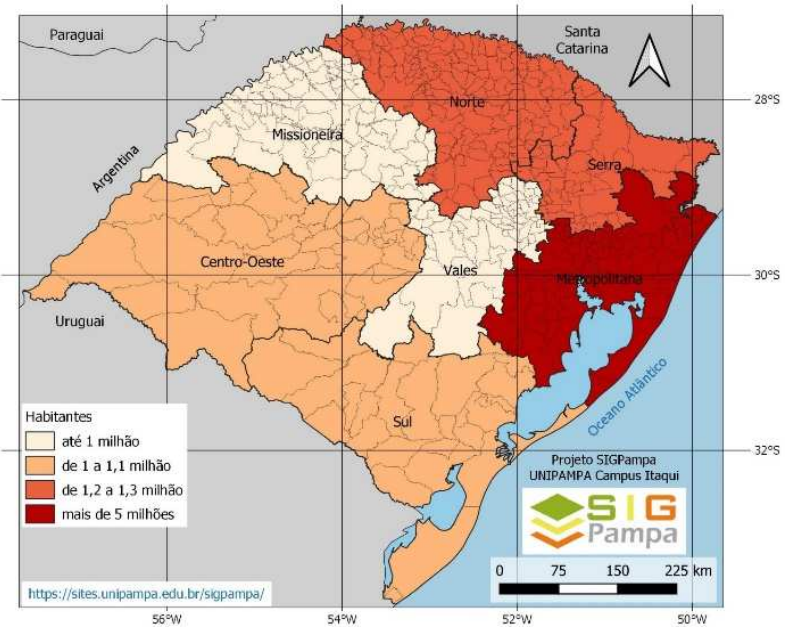

Fonte: elaborado pelos autores a partir de IBGE $(2017,2018,2019)$, SES-RS (2020) e DATASUS (2020)

Figura 4 - Leitos de UTI, do SUS, considerando 0 plano de contingenciamento nível 3, para cada 10.000 habitantes, reservados ao tratamento de pacientes da COVID-19, no Rio Grande do Sul, por Macrorregião de planejamento da saúde

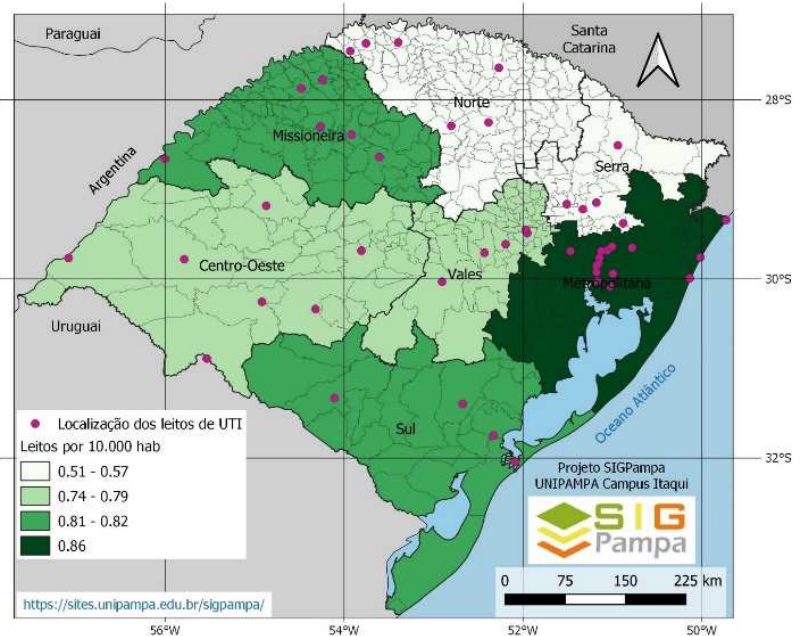

Fonte: elaborado pelos autores a partir de IBGE $(2017,2018,2019)$, SES-RS (2020) e DATASUS (2020)
Figura 3 - Leitos de UTI, do SUS, para cada 10.000 habitantes, antes da pandemia da COVID-19, no Rio Grande do Sul, por Macrorregião de planejamento da saúde

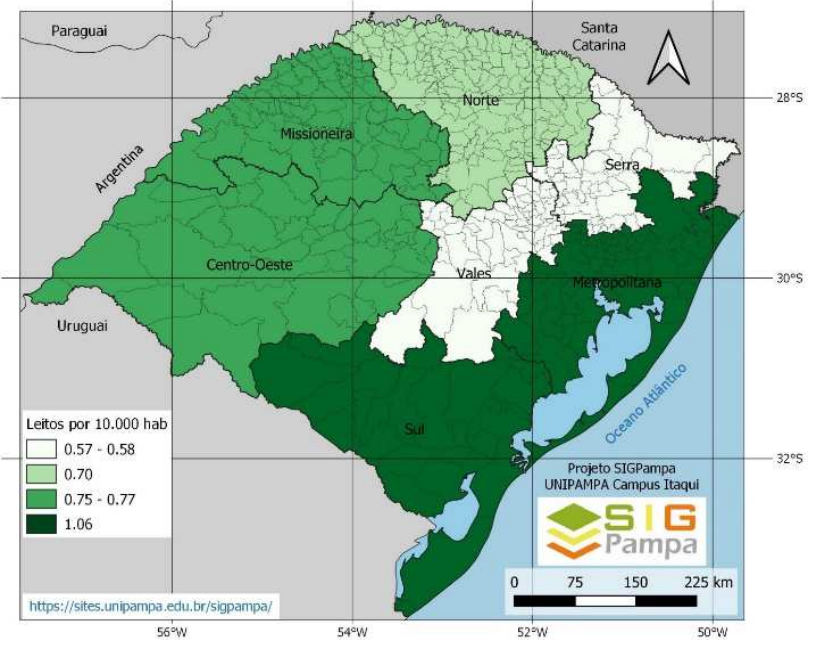

Fonte: elaborado pelos autores a partir de IBGE $(2017,2018,2019)$, SES-RS (2020) e DATASUS (2020)

Figura 5 - Distribuição geográfica e número de ambulâncias de suporte avançado, por Macrorregião de planejamento da saúde, no Rio Grande do Sul

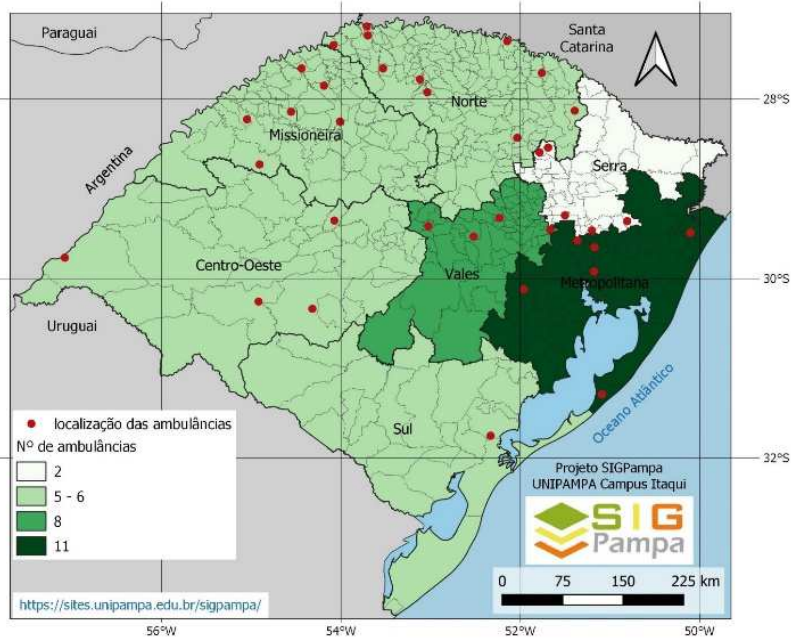

Fonte: elaborado pelos autores a partir de IBGE (2017, 2018, 2019), SES-RS (2020) e DATASUS (2020) 


\section{CONSIDERAÇÕES FINAIS}

Cabe ressaltar que, por se tratar de uma pesquisa em desenvolvimento pelo projeto SIGPampa, do Campus Itaqui da UNIPAMPA, os dados apresentados neste material são passíveis de adequações e atualizações, considerando que o próprio Plano de Contingência Hospitalar do RS (SES-RS, 2020) passa por atualizações em função do avanço dos casos de COVID-19. Contudo, algumas questões podem ser apresentadas no sentido de análises futuras que tenham por objetivo a instrumentalização de políticas públicas que visem melhorar o sistema de saúde no Rio Grande do Sul, a saber:

(i) Qual o critério para a distribuição dos leitos de UTI e das ambulâncias de suporte avançado?

(ii) Tais critérios levam em consideração as reais necessidades da população, em nível de Macrorregião, Coordenadoria Regional e Região de Saúde, como uma política pública de amplo atendimento?

(iii) Como fica, em casos de pandemia, a relação com os centros de referência em saúde (nas mais diversas especialidades)?

(iv) Em todo os mapas ocorre uma predominância dos maiores índices na Macrorregião Metropolitana. Contudo, para as demais macrorregiões, não há um padrão de distribuição que considere a equação leitos por habitantes de forma linear. De que forma isto pode ser equacionado como política pública?

\section{REFERÊNCIAS BIBLIOGRÁFICAS}

AMIB. Comunicado da AMIB sobre o avanço do COVID-19 e a necessidade de leitos em UTIs no futuro. 2020.

Disponível em

http://www.somiti.org.br/arquivos/site/comunicacao/noticias/2020/covid-19/comunicado-daamib-sobre-o-avanco-do-covid-19-e-a-necessidade-de-leitos-em-utis-no-futuro.pdf

BONVIM, C.; MEDEIROS, Z. Epidemiologia e geografia: dos primórdios ao geoprocessamento. Revista Espaço para a Saúde, Londrina, v. 10, n. 1, p. 53-62, dez. 2008.

DATASUS. Cadastro Nacional de Estabelecimentos de Saúde - TABNET. 2020. Disponível em http://www2.datasus.gov.br/DATASUS/index.php?area $=02$

GASS, S. L. B.; DA SILVA, D. M. Estruturação de uma base de dados sobre a COVID-19 no estado do Rio Grande do Sul, Brasil. SocArXiv. 2020. https://doi.org/10.31235/osf.io/5qu73

IBGE. Base cartográfica vetorial contínua do Brasil em escala 1:250.000, versão 2017. Disponível em

http://geoftp.ibge.gov.br/cartas e mapas/bases cartograficas continuas/bc250/versao2017/

IBGE. Malha territorial municipal compatível com a escala 1:250.000, versão 2018. Disponível em http://geoftp.ibge.gov.br/organizacao do territorio/malhas territoriais/malhas municipais/mu nicipio 2018/

IBGE. SIDRA Tabela 6579 - população residente estimada para o ano de 2019. Rio de Janeiro: IBGE, 2019. Disponível em https://sidra.ibge.gov.br/tabela/6579

SES-RS. Organização da saúde no território gaúcho - municípios, CRS, Regiões de Saúde, Macrorregiões e Coredes. Porto Alegre: Secretaria Estadual da Saúde do Rio Grande do Sul, 
2013. Disponível em https://saude.rs.gov.br/upload/arquivos/carga20190107/29150758municipio-regiao-crs-macro-corede.pdf

SES-RS. Plano de Contingência Hospitalar COVID-19 - Abril - versão 9.0, de 07/04/2020. Porto Alegre: Secretaria Estadual da Saúde do Rio Grande do Sul, 2020. Disponível em https://saude.rs.gov.br/upload/arquivos/202004/22175138-contingenciamento-dre-daha-4.pdf

UNIPAMPA. Estudo sobre coronavírus no RS tem primeiros resultados. 17 de abr. de 2020. Disponível em https://sites.unipampa.edu.br/coronavirus/2020/04/17/estudo-sobrecoronavirus-no-rs-tem-primeiros-resultados/\#more-605

WIKIPEDIA. Coronavirus. 2020a. https://pt.wikipedia.org/wiki/Coronav\%C3\%ADrus. Acessado em 30 de mar. 2020.

WIKIPEDIA. COVID-19. 2020b. https://pt.wikipedia.org/wiki/COVID-19. Acessado em 30 de mar. 2020.

WIKIPEDIA. Pandemia de COVID-19. 2020c. https://pt.wikipedia.org/wiki/Pandemia de COVID-19. Acessado em 30 de mar. 2020. 